\title{
BMP signaling pathways affect differently migration and invasion of esophageal squamous cancer cells
}

\author{
MIN HU, FACAI CUI, FENGZHEN LIU, JINLIN WANG, XIAOXIA WEI and YI LI \\ Clinical Biochemistry Laboratory, Medical Diagnostic Department of Henan \\ Provincial People's Hospital, Zhengzhou, Henan 450000, P.R. China
}

Received October 21, 2016; Accepted December 5, 2016

DOI: $10.3892 /$ ijo.2016.3802

\begin{abstract}
Bone morphogenetic proteins (BMPs) are broadly involved in normal embryo development and abnormal pathological process such as cancer. The complexity and diversity of BMPs and their signaling pathways impose quite different or even conflicting effects on clinical traits of tumors. The aim of the present study was to investigate whether different BMPs, including BMP2, BMP4, BMP6 and BMP7, influence esophageal squamous cancer cell (ESCC) growth, invasion and metastasis. BMP6 and type I receptor ALK2 and type II receptor BMPRII, ActRIIA and ActRIIB were expressed in all ESCC cell lines. In addition, adenovirus-mediated BMP overexpression did not affect ECA-109 cell growth. BMP6/7 overexpression increased ECA-109 cell invasion and metastasis, activated SMAD1/5/8 signal pathway and induced downstream gene ID1 expression. While BMP2/4 overexpression reduced ECA-109 cell invasion and metastasis and obviously promoted ERK1/2, P-38 and JNK activation with weak SMAD1/5/8 phosphorylation. When BMP6/7 favorite type I receptor ALK2 or type II receptor BMPRII was interfered with by dominant-negative mutation, BMP6/7-induced invasion and metastasis augmentation disappeared. Further investigation on clinical ESCC samples and non-tumorous adjacent tissue found that tumors with triple-positive BMP6, ALK2 and BMPRII had deeper growth than tumors with only BMP6 expression. These results suggested that different BMPs distinctly affected esophageal squamous cancer cell invasion and metastasis by employing different signal pathways.
\end{abstract}

\section{Introduction}

As a high risk cancer, esophageal cancer possesses several adverse characteristics including difficult early diagnosis, rapid tumor growth and metastasis, relatively low response to

Correspondence to: Dr Min Hu, Clinical Biochemistry Laboratory, Medical Diagnostic Department of Henan Provincial People's Hospital, Zhengzhou, Henan 450000, P.R. China

E-mail:yaushz@126.com

Key words: bone morphogenetic proteins, esophageal squamous cancer cells, invasion and metastasis, ALK2, BMPRII drug treatment and poor 5-years survival. The major type of esophageal cancer in Asian countries is esophageal squamous cell carcinoma (ESCC), while esophageal adenocarcinoma occupies predominant cancer type in Western countries. Although great efforts have been made to explore the mechanisms of epigenetic or genetic modifications occurred during esophageal tumorigenesis, the molecular biology of esophageal cancer is still too complicated to understand throughly. Research on esophageal squamous cancer indicated that activation of oncogenes and impaired signaling pathways, such as c-Myc, VEGF and TGF- $\beta$ (1), actively participated in the development of esophageal cancer, and correspondingly molecular treatments targeted to abnormal signaling pathways were widely designed and tested.

Bone morphogenetic proteins (BMP), as the members of transforming growth factor- $\beta$ (TGF- $\beta$ ) superfamily, were initially described for osteoinductive potentiality. During human development, BMPs also take part in many biological activities including cell proliferation, migration, apoptosis and differentiation (2). As secretory proteins, BMPs exert functions by coupling with receptors. There are two groups of BMP receptors, named type I and II receptors of serine/ threonine kinase. Type I receptors contain activin receptorlike kinase-2, -3 and -6 (ALK-2, ALK3 and ALK-6), while type II receptors include BMPRII, ActRIIA and ActRIIB. By binding to type II receptor and subsequently activated type I receptor, BMPs transduce signaling via phosphorylating SMAD1/5/8 proteins, which further coordinate with co-SMAD4 protein to activate target downstream genes. Except their indispensable roles in normal development, BMPs and their receptors were extensively found abnormally expressed in carcinomas, such as prostate, breast and ovarian cancer (3-5). Studies of those cancers indicated that malfunctions of BMP signaling pathways were more or less responsible for cancer progress, including proliferation, invasion and metastasis and protected cells from death. BMP2 and BMP4 are different from BMP6 and BMP7 based on their structural sequence similarity. BMP2 and BMP4 share $92 \%$ similar amino acid sequence, while BMP6 and BMP7 can be categorized as a subgroup (6,7). BMP2 was found related with metastasis in gastric cancer and melanoma $(8,9)$, proliferation in prostate and lung cancer $(10,11)$, while BMP4 was reported to induce epithelial-to-mesenchymal transition (EMT) in ovarian and pancreatic cancer $(12,13)$. BMP6 showed its roles 
in promoting E-cadherin expression and potential function in breast cancer metastasis (3). BMP7 exhibited its contradictory roles in promoting EMT in melanoma and related with metastasis in breast cancer or inhibiting TGF- $\beta$-induced EMT in esophageal adenocarcinoma $(1,14,15)$. SMAD-mediated BMP signaling pathway predominates in most BMP involved activities, but other signaling pathways have also been reported to be employed or cross-linked with BMPs, such as PI3K/AKT, MAPK/ERK/p38, WNT/ $\beta$-catenin and TGF- $\beta$ (16-18). The flexible usage of BMP receptors and comprehensive signaling pathways may explain the conflicting results of BMP functions in cancers. Recently, Yuen et al (19) found BMP6 expression widely in clinical esophageal cancer samples and strong BMP6 expression associated with faint BMP inhibitor Noggin expression was correlated with shorter survival. BMP7 expression was also reported by Megumi et al (20) to predict poor prognosis of esophageal squamous cells. However, the roles of BMPs in esophageal cancer and underlying mechanisms need to be elucidated for further understanding the value of BMPs in esophageal cancer therapy.

In the present study, we detected BMPs and their receptor expression in ESCC cell lines and explored whether overexpression of BMP2, 4, 6 and 7 would affect ESCC cell proliferation, invasion and metastasis. Distinct influence on invasion and metastasis exhibited by BMP2/4 and BMP6/7 were attributed to different signal pathways. BMP6/7 promoted ECA-109 cell invasion and metastasis through ALK2 and BMPRII-mediated classical SMAD1/5/8dependent pathways while BMP $2 / 4$ reduced invasive and migratory properties of ECA-109 without effective activation of SMAD1/5/8 pathways but simultaneously upregulated phosphorylated P-38, ERK and JNK. Classical BMP signal pathway interference by DN-ALK2 or DN-BMPRII significantly downregulated ECA-109 cell growth, survival, invasion and metastasis. Clinical ESCC patient samples showed different expression pattern of BMP6, ALK2 and BMPRII, but tumors with triple-positive expression of the proteins had deeper invasion. Taken together, this study indicates that BMP6/7 promotes invasion and metastasis of esophageal squamous cancer through ALK2-SMAD pathways, which can serve as potential targets for esophageal squamous cancer therapy.

\section{Materials and methods}

Cell culture and materials. Esophageal squamous cancer cell lines ECA-109, KYSE150 and KYSE180 were maintained in RPMI-1640 medium (HyClone Laboratories, Inc., Logan, UT, USA) supplemented with $10 \%$ fetal bovine serum (FBS; HyClone Laboratories). HEK293 cells used for adenovirus amplification and HCT-116 cells used for adenovirus-expression protein condition medium were cultured in Dulbecco's modified Eagle's medium (DMEM) with 10\% FBS. All cells were maintained with $1 \%$ penicillin/streptomycin in $5 \% \mathrm{CO}_{2}$ at $37^{\circ} \mathrm{C}$. Adenovirus expressing BMP2, BMP4, BMP6, BMP7 and Noggin or dominant-negative mutants of ALK2 and BMPRII were generously offered by professor Tong-Chuan He (Chicago University, Chicago, IL, USA). Ad-GFP served as negative control for the adenovirus involved experiments. Viral titers were determined by infecting 293 cells with gradient dilutions of adenovirus before use. Adenovirus of pfu up to $10^{8}$ were applied, respectively.

RNA extraction and PCR. Total RNA from esophageal cancer cells were obtained by TRIzol reagent (Invitrogen) and chloroform-isopropanol extraction according to the protocol provided by the manufacturer. RNA quantity and integrity were evaluated by NanoDrop 1000 spectrophoto-meter (Thermo Fisher Scientific, Inc., Waltham, MA, USA) and denaturing gel electrophoresis. Two nanogram of total RNA was reverse transcribed and synthesized into cDNA by PrimeScript kit (Takara). Primers used to detect different gene expression and PCR conditions are listed in Table I with GAPDH used as internal control. The analysis of PCR products was performed on Quantity One software (Bio-Rad Laboratories, Hercules, CA, USA).

Western blot analysis. After different infection of Ad-BMPs for $24 \mathrm{~h}$, ESCC cells were serum-starved for $24 \mathrm{~h}$ and RIPA cell lysis buffer (Biyuntian Biotechnology Co., Ltd., Shanghai, China) supplemented with $1 \mathrm{mmol}$ PMSF was added. After centrifuged at $1,3000 \mathrm{xg}$, at $4^{\circ} \mathrm{C}$ for $30 \mathrm{~min}$, protein concentration was determined by NanoDrop 1000 spectrophotometer (Thermo Fisher Scientific). A total of $50 \mu \mathrm{g}$ protein samples were loaded and resolved by $10 \%$ SDS-PAGE. Then protein bands were transferred onto polyvinylidene difluoride membranes (PVDF; Millipore, Billerica, MA, USA). Primary antibody was diluted in TBST buffer (dilution as 1:1,000) and applied on the membrane to incubate at $4^{\circ} \mathrm{C}$ overnight. Horseradish peroxidase-conjugated secondary antibody (Zhongshan Golden Bridge Biotechnology Co., Ltd., Beijing, China) was incubated with PVDF membrane for $1 \mathrm{~h}$ at $37^{\circ} \mathrm{C}$ next day after rinsing by TBST buffer. Then the membrane was extensively washed by TBST and protein bands visualized using chemiluminescence reagent (Pierce) in ChemiDoc ${ }^{\mathrm{TM}}$ $\mathrm{XRS}+$ System (Bio-Rad Laboratories). Primary anti-pSmad1/5/8 (1/5-S453/465 and 8-S462/428), anti-p-Smad1 (S463/465), anti-T-Smad1, anti-p-JNK (T183/185), anti-JNK and anti-p-P38 (T188/Y182) were purchased from Cell Singaling Technology (Danvers, MA, USA). Internal control anti- $\beta$-actin was purchased from Santa Cruz Biotechnology (Santa Cruz, CA, USA).

Cell proliferation assay. Cell proliferation was performed by 3-(4,5-dimethylthiazol-2-yl)-2,5-diphenyl tetrazolium bromide (MTT; Promega, Madison, WI, USA) colorimetric assay. Briefly, esophageal cancer cells were infected by different adenovirus for $24 \mathrm{~h}$ and then seeded into 96-well plate at 5,000 cells/well. After cells tightly attached, MTT reagent was added to detect cell viability as $0 \mathrm{~h}$ time-point or at $24-\mathrm{h}$ interval. For each well, $20 \mu \mathrm{l} 5 \mathrm{mg} / \mathrm{ml}$ MTT reagent was added and incubated at $37^{\circ} \mathrm{C}$ for $4 \mathrm{~h}$. DMSO was used to dissolve formazan precipitation at the end of incubation and then optical density of each well was measured at $490 \mathrm{~nm}$ by a plate reader (Sunrise Remote; Tecan Group Ltd., Männedorf, Switzerland). Each sample contained at least 5-wells and experiments were performed three times.

Invasion and migration assay. To determine ESCC cell invasion and migration, Transwell migration chambers (Corning 
Table I. PCR primers for BMPs and BMP receptors.

\begin{tabular}{|c|c|c|c|}
\hline Primer & Sequence & Temperature $\left({ }^{\circ} \mathrm{C}\right)$ & Product length (bp) \\
\hline ALK1 & $\begin{array}{l}\text { GGCTCCCTCTACGACTTTCT } \\
\text { TGGGCAATGGCTGGTTT }\end{array}$ & 57 & 137 \\
\hline ALK2 & $\begin{array}{l}\text { AGGATTACAAGCCACCG } \\
\text { TACCAGCATTCTTTCATTAG }\end{array}$ & 52 & 163 \\
\hline ALK3 & $\begin{array}{l}\text { TCTTGGAGGAGTCGTAA } \\
\text { GTAAATGTATAGCTGAGGC }\end{array}$ & 50 & 181 \\
\hline ALK6 & $\begin{array}{l}\text { AAATGTGGGCACCAAGAAAGA } \\
\text { ACAGGCAACCCAGAGTCATC }\end{array}$ & 55 & 171 \\
\hline BMP2 & $\begin{array}{l}\text { CCTACATGCTAGACCTGTATCGCA } \\
\text { CACCAACCTGGTGTCCAAAAGT }\end{array}$ & 52 & 362 \\
\hline BMP4 & $\begin{array}{l}\text { CAGCACTGGTCTTGAGTATCCTGA } \\
\text { CGTGTCCAGTAGTCGTGTGATGA }\end{array}$ & 52 & 307 \\
\hline BMP6 & $\begin{array}{l}\text { CGACAACAGAGTCGTAATCG } \\
\text { GCATTCTCCATCACAGTAATTG }\end{array}$ & 52 & 195 \\
\hline BMP7 & $\begin{array}{l}\text { ACGCTTCGACAATGAGACGTTC } \\
\text { TGGCGTTCATGTAGGAGTTCAG }\end{array}$ & 52 & 572 \\
\hline BMPRII & $\begin{array}{l}\text { AAATAGCCTGGCAGTGAG } \\
\text { ATGTGACAGGTTGCGTTC }\end{array}$ & 53 & 196 \\
\hline ActRIIA & $\begin{array}{l}\text { GAAGATGAGGCCCACCATTA } \\
\text { GACAGAGGTCACCAGGGAAA }\end{array}$ & 52 & 179 \\
\hline ActRIIB & $\begin{array}{l}\text { AАACCTGCCATATCTCAC } \\
\text { GCACССТСТААТАССТСТ }\end{array}$ & 52 & 199 \\
\hline ID1 & $\begin{array}{l}\text { CGGTCTCATTTCTTCTCG } \\
\text { TCGGTCTTGTTCTCCCTC }\end{array}$ & 57 & 182 \\
\hline GAPDH & $\begin{array}{l}\text { CAGCGACACCCACTCCTC } \\
\text { TGAGGTCCACCACCCTGT }\end{array}$ & 52 & 120 \\
\hline
\end{tabular}

Incorporated, Corning, NY, USA) with $8 \mu \mathrm{m}$ pores were used. After adenovirus infection for $24 \mathrm{~h}$, cells were trypsinized and seeded at $5 \times 10^{5}$ in duplicate in the upper chamber of Transwell with or without Matrigel (Invitrogen). Lower chamber of 24-well plate contained $20 \%$ FBS. After allowing cell migration for $24 \mathrm{~h}$, the filters of Transwell were dried, fixed and stained by hematoxylin and eosin (H\&E) or crystal violet. Ten random high power fields with evenly distributing cells were chosen to count invaded cells. All experiments were performed at least three times.

Transfection and SMAD-responsive alkaline phosphatase activity detection. Alkaline phosphatase reporter plasmid pSEAP2 (Clontech Laboratories, Inc., Mountain View, CA, USA) containing two BMP response elements (BRE) was constructed previously, named as pSEAP2-BRE. pSEAP2-BRE was transfected into ECA-109 cells using Lipofectamine 2000 reagent (Invitrogen) with conditional medium of GFP or BMPs produced by the HEK293 cells. Supernatant of cells was extracted after transfection for $24 \mathrm{~h}$ and alkaline phosphatase activity was detected by the SEAP chemiluminescence.

Colony formation assay. After ECA-109 infection with AdDN-ALK2 or AdDN-BMPRII for $24 \mathrm{~h}$, low density cells were seeded in 12-well plate by 100 cells/well. At least three wells were employed for each group. ECA-109 and ECA-109 infected by AdGFP served as control. Then cells were incubated in $37^{\circ} \mathrm{C}$ for a week. The plate was washed gently by phosphate-buffered saline (PBS) to remove dead cells and then stained by crystal violet.

ESCC tumor samples. ESCC patients enrolled in this study were the same cohort as a previous report (21). All samples, including tumor tissues and non-cancerous tissues, were collected and kept in liquid nitrogen until RNA extraction. The patient clinical histopathological profiles were also collected and classified according to the differentiation status, TMN stage and tumor depth, which contain shallow tumor invasion within the mucosa and deep submucosal invasion. 
A

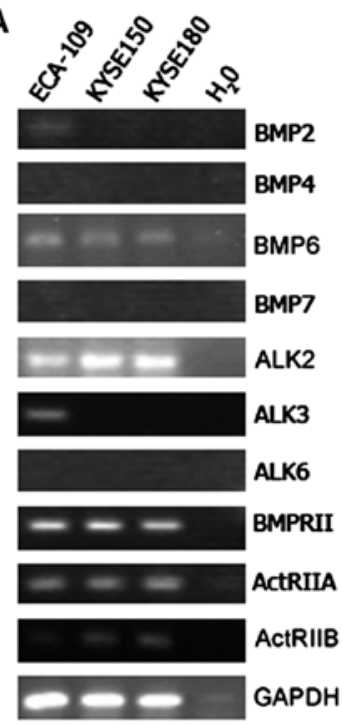

B

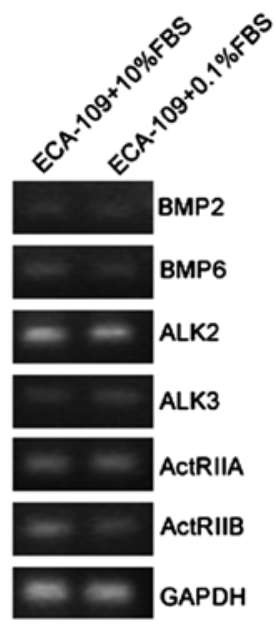

Table II. Transwell assay with or without Matrigel (mean cell numbers/HPS).

\begin{tabular}{ccccccc}
\hline & Matrigel & GFP & BMP2 & BMP4 & BMP6 & BMP7 \\
\hline ECA-109 & - & 52 & 47 & 45 & 69 & 71 \\
& + & 36 & 30 & 32 & 51 & 57 \\
KYSE150 & - & 69 & 21 & 20 & 55 & 47 \\
& + & 33 & 26 & 29 & 41 & 45 \\
KYSE180 & - & 57 & 49 & 45 & 73 & 66 \\
& + & 31 & 24 & 28 & 47 & 45 \\
\hline
\end{tabular}

Overexpression of BMPs has no effect on cell proliferation but differentially affect invasion and metastasis. Expression of BMP receptors in esophageal cancer cell lines lays the foundation that BMP signaling pathway may be potentially viable in ESCC cells. Therefore, different BMPs were overexpressed in ECA-109 cells, respectively, to evaluate their impacts on cells proliferation (Fig. 2A). Compared with GFP control group, none of the BMP overexpression changed the proliferation rates of ESCC cells, while adenovirus-loading effect retarded cell growth relatively (Fig. 2B). Besides, although serum deprivation (0.1\% FBS) slightly depressed ECA-109 proliferation, no difference was observed among BMP-overexpressing groups and control group (Fig. 2C). Many reports have indicated that BMPs extensively participate in cancer-related invasion and metastasis. Therefore, the impact of BMPs on invasion and metastasis of ESCC cells was determined by Transwell chamber assay. Surprisingly, not only overexpression of BMPs significantly influenced ESCC cell invasion and metastasis, but different BMPs showed distinct effects. BMP2/4 expression led to $>20 \%$ reduction of invasion and migration, while BMP6/7 overexpression somehow increased ECA-109 cell invasion and migration by $10-20 \%$ (Fig. 3). Similar results were also found in KYSE150 and KYSE180 cell lines (Table II). evaluate the functions of BMP signaling pathways in ESCC, RT-PCR analysis to detect mRNA transcripts for BMP ligands (BMP2, 4, 6 and 7), type I receptors (ALK1, 2, 3 and $6)$ and type II receptors (BMPRII, ActRIIA and ActRIIB) was performed. As shown in Fig. 1A, transcripts of ALK2, BMPRII, ActRIIA and ActRIIB were positive in all three ESCC cell lines, ECA-109, KYSE150 and KYSE180. None of them expressed type I receptor ALK6. BMP6 expression was detected in all these cell lines, consistent with the previous report of Yuen et al (19) that BMP6 can be detected in most of esophageal squamous cells and clinical esophageal cancer tissues. We also found weak expression of BMP2 in ECA-109 cells. However, BMP4 and BMP7 showed negative expression in three ESCC cell lines.

In some reports, serum concentration is seemingly an important factor which greatly affects BMPs expression and their functions. To address this question, we carried out RT-PCR to detect BMPs and their receptors expression levels under the condition of 10 or $0.1 \%$ FBS supplement for $36 \mathrm{~h}$. No detectable difference was observed in the expression of BMP2, BMP6, ALK2, ALK3, BMPRII, ActRIIA and ActRIIB in ECA-109 cells (Fig. 1B).

BMPs can differently activate Smad1/5/8 signal pathway. Most of BMPs exert their functions by activating classical Smad1/5/8-mediated pathway which lead to transcriptional activation of downstream genes. Total protein was extracted under different BMP overexpression conditions to detect the activation of various signal pathways by western blot analysis. Compared with control, the phosphorylated form Smad1/5/8 was significantly induced under BMP6 (1.39-fold) and BMP7 (1.56-fold) stimulation while slight increment can be seen in BMP2 (1.18-fold) and BMP4 (1.22-fold) groups. Total Smad1/5/8 showed equivalent expression in all groups. There was no detectable expression of p-Smad1 in ECA-109 cells treated either by Ad-GFP or Ad-BMPs, although total Smad1 can be clearly and prevalently observed (Fig. 4A). If BMP-Smad1/5/8 signal pathway is functionally transduced, downstream target genes containing BMP response element in promoter region can be transcriptionally activated accordingly. ID1, as a well-known BMP-target gene, was detected by PCR after BMP overexpression in ECA-109 for $48 \mathrm{~h}$. ID1 mRNA was significantly upregulated by treatment with 
A

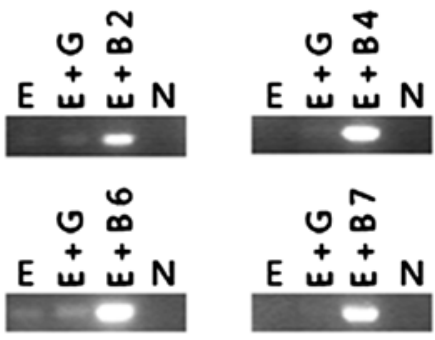

C

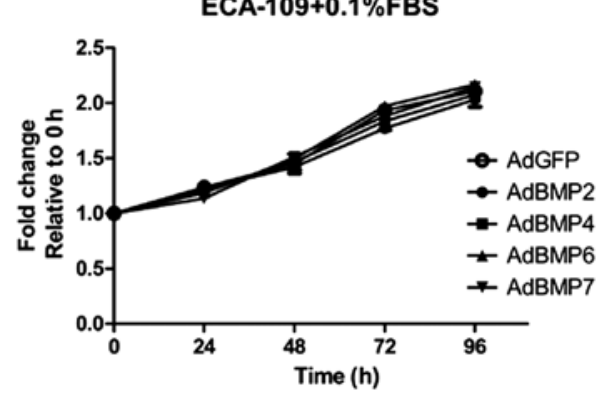

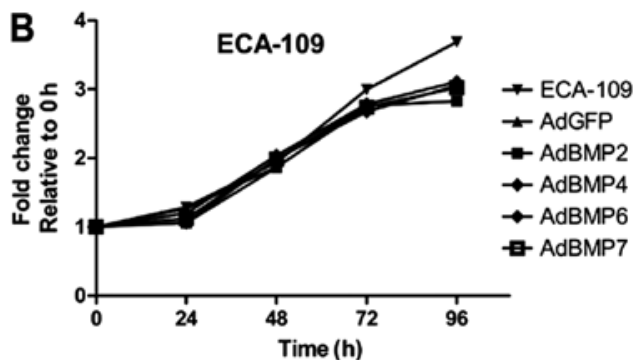
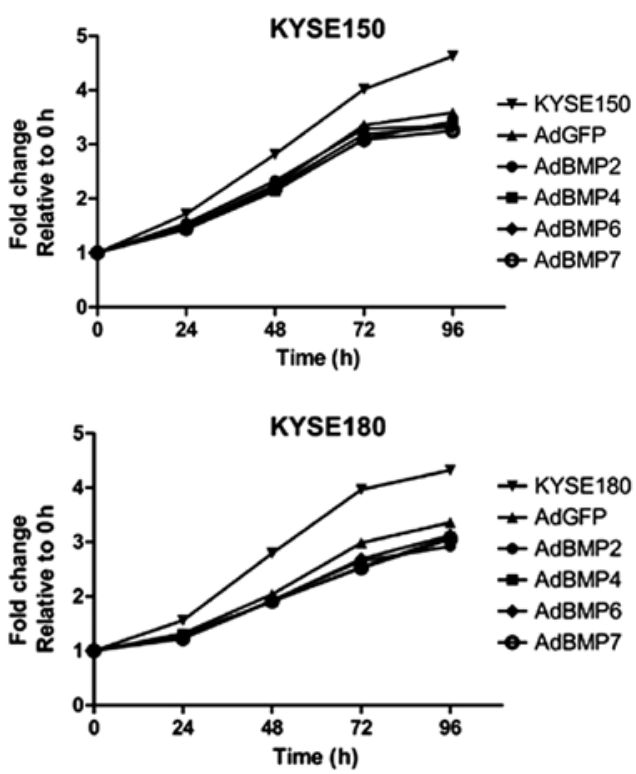

Figure 2. BMP overexpression and their impact on ESCC cell proliferation. Adenovirus-loaded overexpression of BMPs (Ad-BMP) was used to infect ESCC cells, with Ad-GFP served as control. The infected cells showed distinct intense fluorescence at $48 \mathrm{~h}$ after adenovirus infection. (A) Eca-109 cells infected with different Ad-BMPs were subjected to extract total RNA at $48 \mathrm{~h}$ for PCR detection of BMP expression. E, ECA-109; G, GFP; B2, BMP2; B4, BMP4; B6, BMP6; B7, BMP7. (B) Proliferation curve was determined in three cell lines infected with different Ad-BMPs at different time-points. (C) ECA-109 cells infected with different Ad-BMPs were maintained in $0.1 \%$ serum containing medium for $0-96 \mathrm{~h}$ to compare proliferation changes among groups.
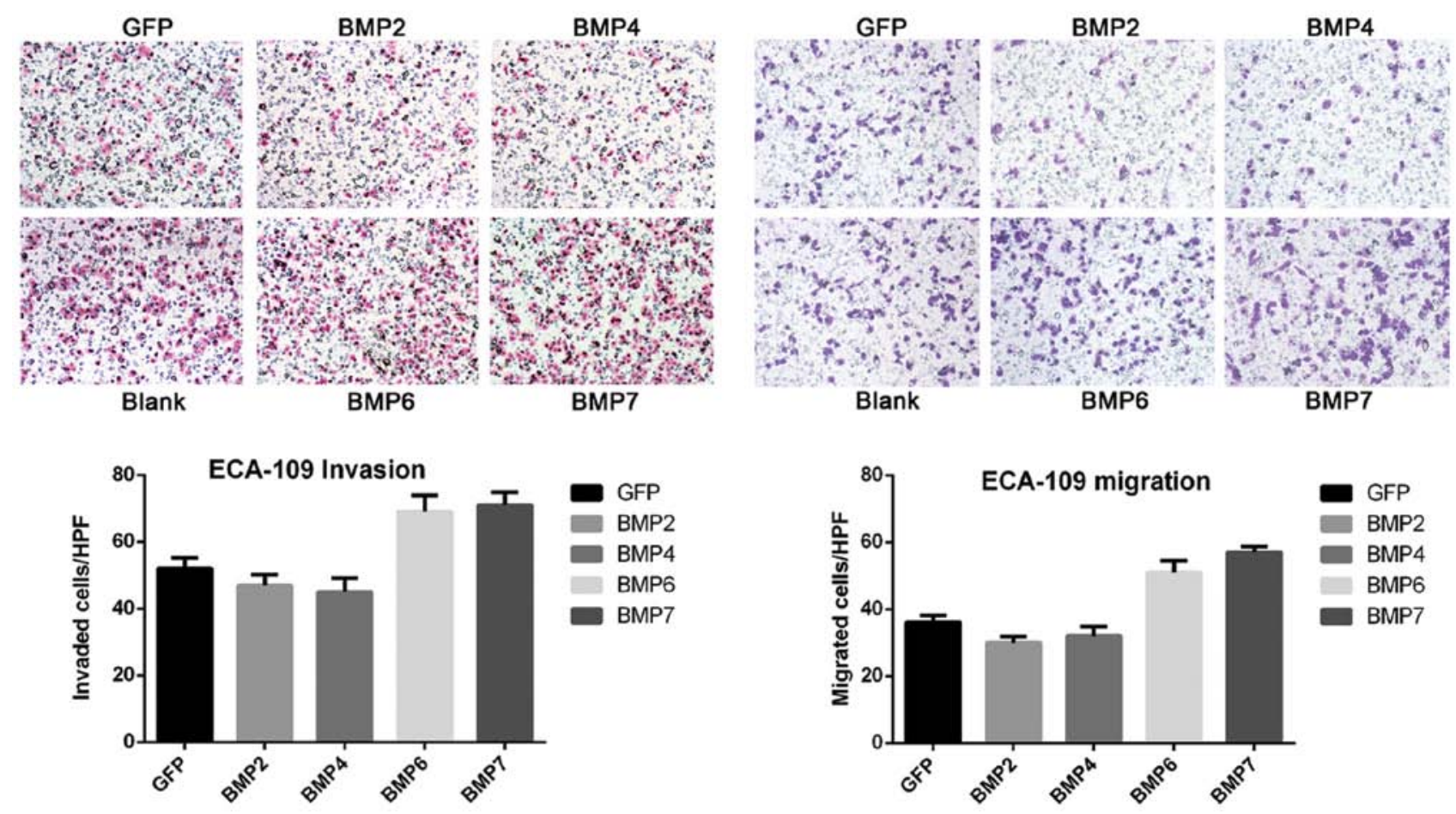

Figure 3. BMP overexpression affects ESCC invasion and metastasis discriminatingly. ESCC cells were infected with different Ad-BMPs for 24 h and then transplanted into upper chamber of Transwell device. Another $24 \mathrm{~h}$ was allowed for cell movement from upper chamber to lower chamber. Transwell with or without Matrigel was designed to detect cell invasive or metastatic ability (left and right part of the figure, respectively). 
A

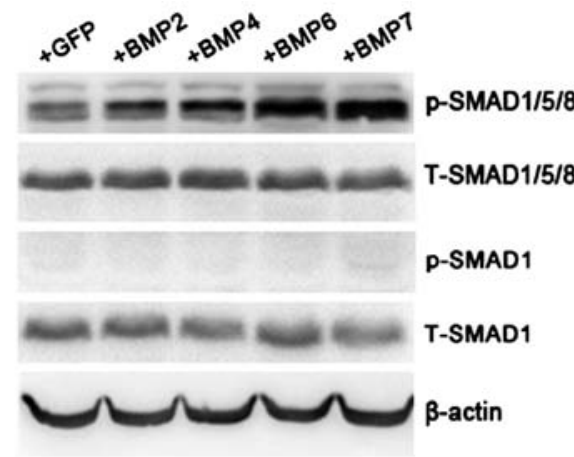

C

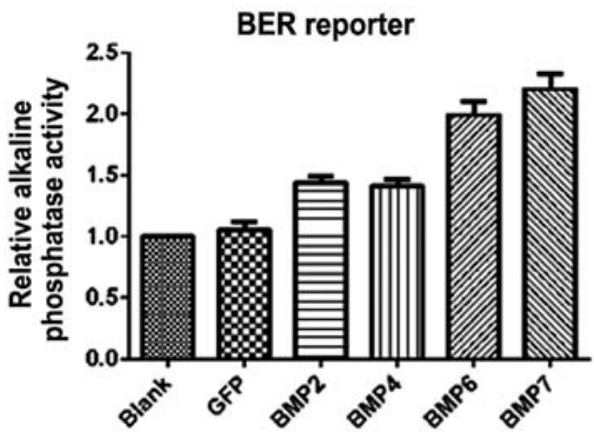

B

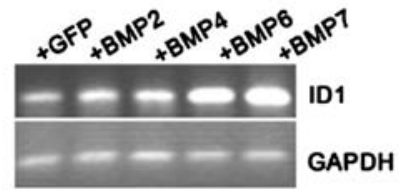

D

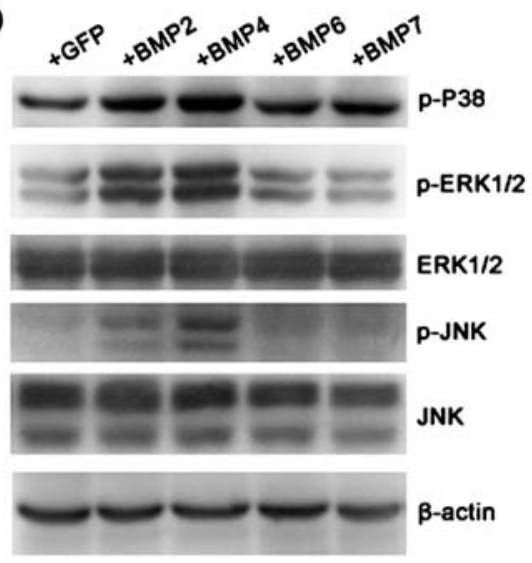

Figure 4. BMP activated distinct signaling pathways. (A) After cells were infected by different Ad-BMPs or Ad-GFP for $48 \mathrm{~h}$, total protein was extracted to detect phosphorylated form and total amount of SMAD1/5/8 and SMAD1. $\beta$-actin served as loading control. (B) BMP classical SMAD1/5/8 signaling pathway downstream target gene transcript ID1 expression was detected by PCR, under different conditions. (C) Alkaline phosphatase activity was detected and compared among different groups. (D). Phosphorylated forms of P-38, ERK and JNK were detected in ECA-109 cells infected with different BMPs for $48 \mathrm{~h}$.

Table III. Clinical characteristics and BMP2, ALK2 and BMPRII expression pattern.

\begin{tabular}{|c|c|c|c|}
\hline Clinical histopathological parameters & $\mathrm{BMP6}^{+} \mathrm{ALK}^{+} \mathrm{BMPRII}^{+}(\mathrm{n}=7)$ & BMP6 $^{+}$ALK2-BMPRII $(\mathrm{n}=26)$ & P-value \\
\hline Histopathologic grading & & & 0.244 \\
\hline Well differentiation & 1 & 11 & \\
\hline Moderate differentiation & 3 & 9 & \\
\hline Poor differentiation & 3 & 6 & \\
\hline Lymphatic invasion & & & 0.080 \\
\hline Negative & 2 & 17 & \\
\hline Positive & 5 & 9 & \\
\hline Tumor depth & & & $0.022^{\mathrm{a}}$ \\
\hline Shallow & 3 & 22 & \\
\hline Deep & 4 & 4 & \\
\hline
\end{tabular}

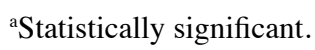

BMP6 (2.12-fold) or BMP7 (2.17-fold) but slightly increased in BMP2 and BMP4 overexpressing cells (Fig. 4B). BMP response element (BRE) alkaline phosphatase reporter was then employed to verify Smad-dependent signaling activation. Alkaline phosphatase activity was obviously high in BMP6/7treated cells (1.9- and 2.2-fold, respectively) compared with control, but mildly increased in BMP2/4-treated cells (1.38- and 1.4-fold; Fig. 4C). Those results indicated that BMP6/7 can significantly activate the downstream signaling pathway in Smad-dependent manner.

BMP2/4 can activate MAPK and JNK pathways. In addition to classical SMAD1/5/8 pathway, BMPs have been reported employing other signaling pathways to exert different functions 
A

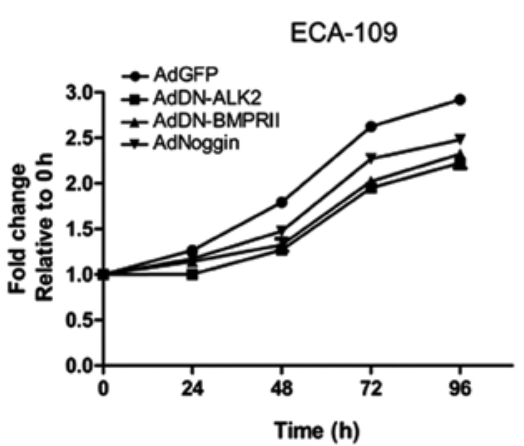

C
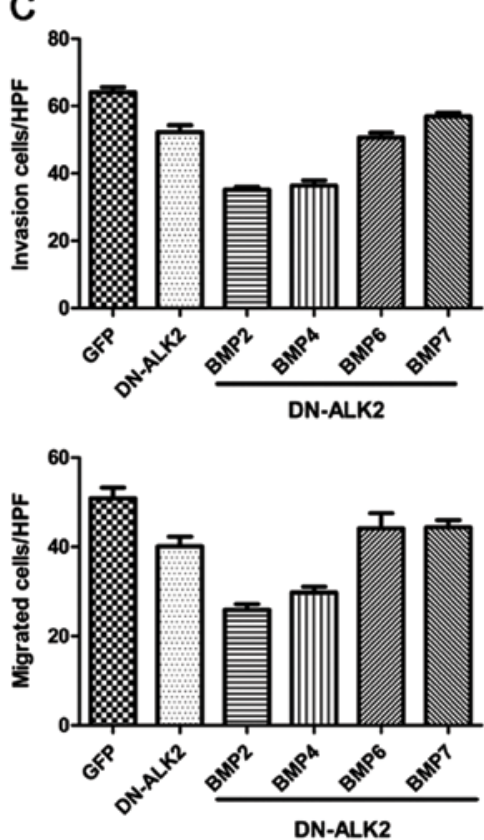

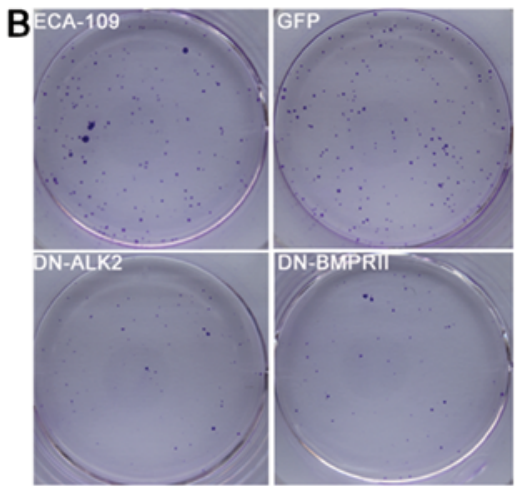

D
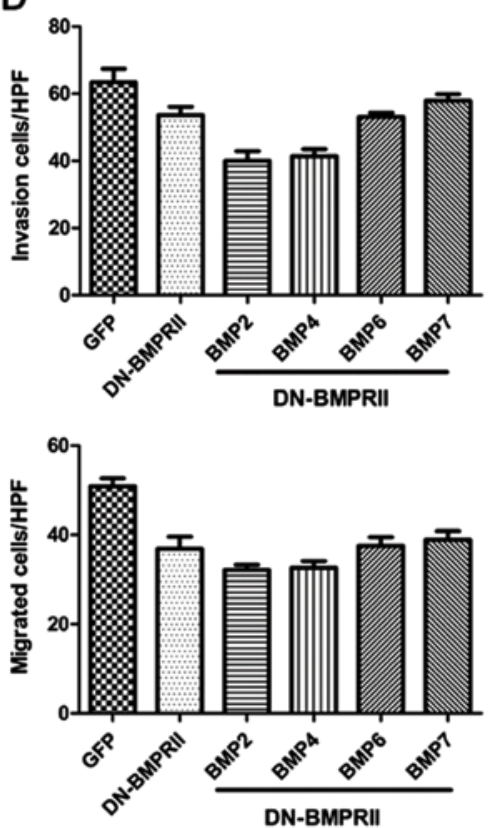

Figure 5. The involvement of BMP type I receptor ALK2 and type II receptor BMPRII in BMP6/7-induced ESCC cell invasion and metastasis. (A) The proliferation curves were determined by MTT assay in ECA-109 cells infected by Ad-GFP, AdDN-ALK2, AdDN-BMPRII and Ad-Noggin, respectively. (B) Colony formation of ECA-109 cells was determined by seeding infected cells in 12-well plate for a week and stained by crystal violet. (C and D) DN-ALK2 or DN-BMPRII condition medium was made and added into adenovirus-infected ECA-109 cells. BMP6 and BMP7-induced ESCC cells invasion and migration were tested by Transwell assay.

in cell biology along with embryo development. Therefore, we explored whether the phenomena also existed in ESCC cells. As shown in Fig. 4D, phosphorylated P-38, ERK1/2 and JNK were obviously upregulated in BMP2- and BMP4-treated groups, but not in BMP6- and BMP7-treated groups.

ALK2 and BMPRII participates in BMP6/7-induced cell invasion and metastasis. Because of sequence homology, BMP6 and BMP7 have similar preference to employ type I receptor ALK2 and type II receptor BMPRII for signal transduction. The classical Smad-mediated pathway activation, and coincidently increased cell invasion as well as metastatic properties prompted us to detect whether ALK2 and BMPRII involved in BMP6/7 overexpression-related events. Adenovirus expressing DN-ALK2 and DN-BMPRII were used, respectively. As demonstrated in Fig. 5A, DN-ALK2 or DN-BMPRII supplement by condition medium can significantly inhibit cell proliferation by $\sim 15-20 \%$ in serum-containing medium $(10 \%$ FBS). BMP inhibitor Noggin served as positive control which decreased cell growth by $10 \%$. Colony survival assay also demonstrated that ECA-109 cells containing DN-ALK2 or DN-BMPRII exhibited slower grow th and less colony formation (Fig. 5B). Then, BMP-overexpressing esophageal cancer cell invasion and metastasis were tested with or without DN-ALK2 or DN-BMPRII interference. As showed in Fig. 5C and D, relative to control, DN-ALK2 or DN-BMPRII expressing cells showed markedly reduced cell number invaded or migrated through Transwell chamber membranes and further halted the increment of invasive and migratory properties induced by BMP6/7 overexpression. However, DN-ALK2 or DN-BMPRII hardly altered the negative effects of BMP2/4 overexpression on invasion and metastasis.

ALK2, BMPRII and BMP6 have different expression pattern in ESCC patients. We used RT-PCR to detect ALK2, BMPRII and BMP6 mRNA expression, respectively, in ESCC tumor samples and adjacent non-cancerous tissues $(n=50)$. Compared to the respective non-cancerous tissue, BMP6 overexpression was broadly observed in 40 out of 50 samples (90\%), while 17 samples showed positive ALK2 (34\%) and 15 samples 
showed BMPRII expression (30\%). Only 7 samples have triple-expression of ALK2, BMPRII and BMP6. Analyzing expression pattern and clinical histopathological features between $\mathrm{BMP}^{+}{ }^{+} \mathrm{ALK}^{+}{ }^{+} \mathrm{BMPRII}^{+}$group and $\mathrm{BMP}^{+} \mathrm{ALK} 2$ BMPRII' samples indicated that positive ALK2 and BMPRII expression contributed to deeper tumor invasion on the background BMP6 expression (Table III), although no effect on tumor differentiation status and lymphatic invasion was observed.

\section{Discussion}

BMPs family contains more than twenty identified members, which are widely involved in multiply biological processes, especially in bone formation and embryo development. For decades, the diverse roles of BMPs in tumor cell growth, invasion, metastasis and angiogenesis have been extensively studied. BMPs and BMP receptors were found in many cancers, including prostate, breast, ovarian, melanoma, lung and esophageal cancer (22-24). Recently, Yuen et al (19) found that BMP6 expression can be detected in the majority of esophageal squamous cancer cell lines and clinical esophageal cancer tissues correlated with tumor progression and prognosis along with BMP antagonist expression level. In this study, BMP6 expression in all three esophageal squamous cancer cell lines and BMP type I and type II receptors can also be detected, indicating that BMP signal would be potentially functional in esophageal cancer cells. BMP7 expression has also been detected in more than half of clinical esophageal squamous cell carcinoma samples and patients with BMP7 expression had deeper invasion and poorer prognosis (20). We did not find any BMP7 expression in esophageal squamous carcinoma cell lines and in clinical ESCC tumors. To investigate the roles BMPs played in esophageal squamous carcinoma, overexpression of different BMPs by adenovirusbased gene expressing were carried out in ECA-109 cells. Although many reports have demonstrated that upregulation of BMPs can greatly affect cancer cell proliferation such as prostate, breast and lung cancer, overexpression of BMP2, 4, 6 and 7 in this study did not affect proliferation rate. One possible reason is that previous reports always employed recombinant human BMP proteins at relatively high concentrations. Such as in the study of Kim et al (25), they found that exogenous recombinant human BMP2 could decrease ESCC cell proliferation in vitro and reduce tumor growth volume in vivo, possibly through Hippo signaling pathways, in which the concentration of rhBMP 2 ranged from $10 \mathrm{nM}$ to $10 \mu \mathrm{M}$ and $10 \mu \mathrm{M}$ was used throughout the research. Kokorina et al (26) also utilized $100 \mathrm{ng} / \mathrm{ml} \mathrm{rhBMP} 2$ to test oral carcinoma cell invasive ability. Several reports showed that the concentration of FBS can greatly affect tumor cell proliferation or growth suppression under BMP supplement conditions. According to this, conflicting results were observed among BMP research on the same tumor type. However, no difference of growth rate or alteration of BMPs and their receptors expression can be found cultured with 10 or $0.1 \%$ FBS here. The effects of serum concentration on BMPs in different cancers provided a notion that the conclusion of the roles of BMPs in cancer should be made depending on the cancer type itself. Therefore, it is possible that the influence of serum concentration is too delicate to be detectable in esophageal squamous carcinoma.

Although the investigation on esophageal squamous tumor clinical samples showed inconsistent upregulation of BMP6, ALK2 was prevalently detected in both esophageal cancer tissues (34\%) and in esophageal cancer cell lines. ALK2 expression may confer a favorable condition for BMP6 binding and promoting esophageal cancer cell motivation. As indicated in other research that BMPs exert biological functions by classical SMAD-mediated pathway, we found that BMP6/7 overexpression significantly activated Smad1/5/8 signaling pathways and then transcriptionally upregulated ID1 expression and BRE-driven alkaline phosphatase reporter activity. Classical Smad-dependent pathway activation has been implicated involved in BMP-induced invasion and metastasis $(27,28)$. ID1 transcriptional activation alone or induced by BMPs was found related with increased cancer aggressiveness (18,29-31). Furthermore, interference of type I receptor ALK2 or type II receptor BMPRII by dominant negative mutants decreased cancer cells invasion or migration through membrane with or without Matrigel. Besides, ALK2 interference or BMP inhibitor Noggin supplement also reduced cell growth and long period clonal survival ability. Combined with the report by Yuen et al (29) that widely expressed BMP6 and low level of BMP antagonists were demonstrated in esophageal cancer, our results suggested that esophageal squamous cancer cell may acquire favorable cell proliferation partly through a BMP6-ALK2 autocrine manner, similar to BMP9 in ovarian cancer.

Notably, BMP2 and BMP4 overexpression somehow reduced cell invasion and metastasis. Compared to BMP6 and BMP7, BMP2 and BMP4 induced less Smad1/5/8 phosphorylation and correspondingly lower BRE alkaline phosphatase reporter activation and ID1 mRNA expression. Additionally, DN-ALK2 or DN-BMPRII exerted much less effect on BMP $2 / 4$ related invasive and migratory events than BMP6/7 exhibited, indicating that BMP-SMAD pathway may not be effective in BMP2 and BMP4 overexpression cases. This hypothesis in turn explained why BMP2 and BMP4 did not enhance cell invasiveness and migration as BMP6 and BMP7 showed. Lavery et al (32) reported that BMP2/4 and BMP6/7 utilized different receptors to induce human osteoblastic differentiation. Whether the similar mechanisms also existing in cancer biology is unknown, but ALK2 interference did not affect BMP2/4-induced activities and BMP2/4 preferential receptor ALK6 was absent. Further investigation on involving signal pathways in BMP2/4-overexpressed cells showed activation of ERK1/2 and P-38 pathways which were nearly unchanged in BMP6/7-overexpressed cells compared with control. The utilization of other signaling pathways by BMPs to exert different functions other than classical SMAD1/5/8 pathways has been addressed in other studies. Jung et al (33) reported that BMP9 potently inhibited liver cancer stem cell percentage by phosphorylating P-38 which in turn activated ID3 expression and p21 induction. In blood vessel endothelium cells, BMP4 induced capillary sprouting relying on ERK phosphorylation activation (34). Although several reports have indicated potential crosstalk between BMPs and MAPK signal pathway, the definite mechanisms is largely unknown. Our reports showed that MAPK signal 
pathway may participate in BMP2/4-related biological activities in esophageal squamous cancer cells. Whether this phenomenon prevalently exists or is tissue- specific need further investigation. The discrepancy of BMP $2 / 4$ and BMP6/7 effects on ESCC cells may reflect their different functions in esophageal development. In normal tissue and organ development, stem cell differentiation and self-renewal ability are strictly regulated. The similarities between cancer stem cells and normal stem cells indicate the complicated tumor traits at differentiation or dedifferentiation perspective. As shown in the study of Jiang et al (35), normal adult mouse esophagus basal progenitor cells resisted BMP4-induced squamous differentiation by expressing BMP inhibitor follistatin, and human immortal esophageal progenitor cells can also be induced to differentiate by BMP4 and proliferation inhibition. BMP4 can also promote hepatic cancer stem cells to differentiation by activating ERK1/2 pathway (36). On the contrary, BMP6 expression in esophageal cancer was negatively related with tumor differentiation and keratinization (7).

The present study demonstrated that BMP signaling is potentially activated in esophageal squamous cancer cells and related with cancer cell growth, invasion and metastasis. BMP6 were shown prevalently expressed in esophageal squamous cancer tissues and cell lines and associated with cancer progress and prognosis (19). BMP6/7 activated SMADdependent classical BMP signaling pathways through type I receptor ALK2. ALK2 interference diminished cell growth, and BMP6/7-induced accelerated invasion and metastasis. Therefore, targeting BMP6/7 signaling pathway in esophageal squamous cancer, such as ALK2-specific inhibitor application, lay a rational foundation for esophageal squamous cancer treatment.

\section{References}

1. Rees JR, Onwuegbusi BA, Save VE, Alderson D and Fitzgerald RC: In vivo and in vitro evidence for transforming growth factor-beta1mediated epithelial to mesenchymal transition in esophageal adenocarcinoma. Cancer Res 66: 9583-9590, 2006.

2. Chen D, Zhao M and Mundy GR: Bone morphogenetic proteins. Growth Factors 22: 233-241, 2004.

3. Yang S, Du J, Wang Z, Yuan W, Qiao Y, Zhang M, Zhang J, Gao S, Yin J, Sun B, et al: BMP-6 promotes E-cadherin expression through repressing deltaEF1 in breast cancer cells. BMC Cancer 7: 211, 2007.

4. Shepherd TG and Nachtigal MW: Identification of a putative autocrine bone morphogenetic protein-signaling pathway in human ovarian surface epithelium and ovarian cancer cells. Endocrinology 144: 3306-3314, 2003.

5. Kim IY, Lee DH, Ahn HJ, Tokunaga H, Song W, Devereaux LM, Jin D, Sampath TK and Morton RA: Expression of bone morphogenetic protein receptors type-IA, -IB and -II correlates with tumor grade in human prostate cancer tissues. Cancer Res 60: 2840-2844, 2000.

6. Celeste AJ, Iannazzi JA, Taylor RC, Hewick RM, Rosen V, Wang EA and Wozney JM: Identification of transforming growth factor beta family members present in bone-inductive protein purified from bovine bone. Proc Natl Acad Sci USA 87: 9843-9847, 1990.

7. Ebisawa T, Tada K, Kitajima I, Tojo K, Sampath TK, Kawabata M, Miyazono K and Imamura T: Characterization of bone morphogenetic protein-6 signaling pathways in osteoblast differentiation. J Cell Sci 112: 3519-3527, 1999.

8. Park Y, Kim JW, Kim DS, Kim EB, Park SJ, Park JY, Choi WS, Song JG, Seo HY, Oh SC, et al: The bone morphogenesis protein-2 (BMP-2) is associated with progression to metastatic disease in gastric cancer. Cancer Res Treat 40: 127-132, 2008.
9. Rothhammer T, Poser I, Soncin F, Bataille F, Moser M and Bosserhoff AK: Bone morphogenic proteins are overexpressed in malignant melanoma and promote cell invasion and migration. Cancer Res 65: 448-456, 2005.

10. Langenfeld EM, Calvano SE, Abou-Nukta F, Lowry SF, Amenta P and Langenfeld $\mathrm{J}$ : The mature bone morphogenetic protein-2 is aberrantly expressed in non-small cell lung carcinomas and stimulates tumor growth of A549 cells. Carcinogenesis 24: 1445-1454, 2003

11. Ide H, Yoshida T, Matsumoto N, Aoki K, Osada Y, Sugimura T and Terada M: Growth regulation of human prostate cancer cells by bone morphogenetic protein-2. Cancer Res 57: 5022-5027, 1997.

12. Thériault BL, Shepherd TG, Mujoomdar ML and Nachtigal MW: BMP4 induces EMT and Rho GTPase activation in human ovarian cancer cells. Carcinogenesis 28: 1153-1162, 2007.

13. Milano F, van Baal JW, Buttar NS, Rygiel AM, de Kort F, DeMars CJ, Rosmolen WD, Bergman JJ, VAn Marle J, Wang KK, et al: Bone morphogenetic protein 4 expressed in esophagitis induces a columnar phenotype in esophageal squamous cells. Gastroenterology 132: 2412-2421, 2007.

14. Na YR, Seok SH, Kim DJ, Han JH, Kim TH, Jung H, Lee BH and Park JH: Bone morphogenetic protein 7 induces mesenchymalto-epithelial transition in melanoma cells, leading to inhibition of metastasis. Cancer Sci 100: 2218-2225, 2009.

15. Alarmo EL, Korhonen T, Kuukasjärvi T, Huhtala H, Holli K and Kallioniemi A: Bone morphogenetic protein 7 expression associates with bone metastasis in breast carcinomas. Ann Oncol 19: 308-314, 2008.

16. Lim M, Chuong CM and Roy-Burman P: PI3K, Erk signaling in BMP7-induced epithelial-mesenchymal transition (EMT) of PC-3 prostate cancer cells in 2- and 3-dimensional cultures. Horm Cancer 2: 298-309, 2011.

17. Chen X, Liao J, Lu Y, Duan X and Sun W: Activation of the PI3K/ Akt pathway mediates bone morphogenetic protein 2-induced invasion of pancreatic cancer cells Panc-1. Pathol Oncol Res 17: 257-261, 2011.

18. Augeri DJ,Langenfeld E, Castle M, Gilleran JA and Langenfeld J: Inhibition of BMP and of TGF $\beta$ receptors downregulates expression of XIAP and TAK1 leading to lung cancer cell death. Mol Cancer 15: 27, 2016.

19. Yuen HF, McCrudden CM, Grills C, Zhang SD, Huang YH, Chan KK, Chan YP, Wong ML, Law S, Srivastava G, et al: Combinatorial use of bone morphogenetic protein 6 , noggin and SOST significantly predicts cancer progression. Cancer Sci 103: 1145-1154, 2012.

20. Megumi K, Ishigami S, Uchikado Y, Kita Y, Okumura H, Matsumoto M, Uenosono Y, Arigami T, Kijima Y, Kitazono M, et al: Clinicopathological significance of BMP7 expression in esophageal squamous cell carcinoma. Ann Surg Oncol 19: 2066-2071, 2012.

21. Hu M, Liu Q, Song P, Zhan X, Luo M, Liu C, Yang D, Cai Y, Zhang F, Jiang F, et al: Abnormal expression of the mitotic checkpoint protein BubR1 contributes to the anti-microtubule drug resistance of esophageal squamous cell carcinoma cells. Oncol Rep 29: 185-192, 2013.

22. Pickup MW, Hover LD, Guo Y, Gorska AE, Chytil A, Novitskiy SV, Moses HL and Owens P: Deletion of the BMP receptor BMPR1a impairs mammary tumor formation and metastasis. Oncotarget 6: 22890-22904, 2015.

23. Langenfeld E, Hong CC, Lanke G and Langenfeld J: Bone morphogenetic protein type I receptor antagonists decrease growth and induce cell death of lung cancer cell lines. PLoS One 8: e61256, 2013.

24. Wahdan-Alaswad RS, Song K, Krebs TL, Shola DT, Gomez JA, Matsuyama S and Danielpour D: Insulin-like growth factor I suppresses bone morphogenetic protein signaling in prostate cancer cells by activating mTOR signaling. Cancer Res 70: 9106-9117, 2010

25. Kim SM, Ye S, Rah SY, Park BH, Wang H, Kim JR, Kim SH, Jang KY and Lee KB: RhBMP-2 activates hippo signaling through RASSF1 in esophageal cancer cells. Sci Rep 6: 26821, 2016 .

26. Kokorina NA, Zakharkin SO, Krebsbach PH and Nussenbaum B: Treatment effects of rhBMP-2 on invasiveness of oral carcinoma cell lines. Laryngoscope 121: 1876-1880, 2011.

27. Peng J, Yoshioka Y, Mandai M, Matsumura N, Baba T, Yamaguchi K, Hamanishi J, Kharma B, Murakami R, Abiko K, et al: The BMP signaling pathway leads to enhanced proliferation in serous ovarian cancer-A potential therapeutic target. Mol Carcinog 55: 335-345, 2016. 
28. Chen JC, Yang ST, Lin CY, Hsu CJ, Tsai CH, Su JL and Tang CH: BMP-7 enhances cell migration and $\alpha \mathrm{v} \beta 3$ integrin expression via a c-Src-dependent pathway in human chondrosarcoma cells. PLoS One 9: e112636, 2014.

29. Yuen HF, Chan YP, Chan KK, Chu YY, Wong ML, Law SY, Srivastava G, Wong YC, Wang X and Chan KW: Id-1 and Id-2 are markers for metastasis and prognosis in oesophageal squamous cell carcinoma. Br J Cancer 97: 1409-1415, 2007.

30. Hao J, Lee R, Chang A, Fan J, Labib C, Parsa C, Orlando R, Andresen B and Huang Y: DMH1, a small molecule inhibitor of BMP type i receptors, suppresses growth and invasion of lung cancer. PLoS One 9: e90748, 2014.

31. Herrera B, van Dinther M, Ten Dijke P and Inman GJ: Autocrine bone morphogenetic protein-9 signals through activin receptorlike kinase-2/Smad1/Smad4 to promote ovarian cancer cell proliferation. Cancer Res 69: 9254-9262, 2009.

32. Lavery K, Swain P, Falb D and Alaoui-Ismaili MH: BMP-2/4 and BMP-6/7 differentially utilize cell surface receptors to induce osteoblastic differentiation of human bone marrow-derived mesenchymal stem cells. J Biol Chem 283: 20948-20958, 2008.
33. Jung JW, Yoon SM, Kim S, Jeon YH, Yoon BH, Yang SG, Kim MK, Choe S and Kuo MM: Bone morphogenetic protein-9 is a potent growth inhibitor of hepatocellular carcinoma and reduces the liver cancer stem cells population. Oncotarget: Sep 16, 2016 (Epub ahead of print) doi: 10.18632/oncotarget.12062.

34. Zhou Q, Heinke J, Vargas A, Winnik S, Krauss T, Bode C, Patterson $C$ and Moser M: ERK signaling is a central regulator for BMP-4 dependent capillary sprouting. Cardiovasc Res 76: 390-399, 2007

35. Jiang M, Ku WY, Zhou Z, Dellon ES, Falk GW, Nakagawa H, Wang ML, Liu K, Wang J, Katzka DA, et al: BMP-driven NRF2 activation in esophageal basal cell differentiation and eosinophilic esophagitis. J Clin Invest 125: 1557-1568, 2015.

36. Zhang L, Sun H, Zhao F, Lu P, Ge C, Li H, Hou H, Yan M, Chen T, Jiang G, et al: BMP4 administration induces differentiation of $\mathrm{CD} 33^{+}$hepatic cancer stem cells, blocking their contributions to hepatocellular carcinoma. Cancer Res 72: 4276-4285, 2012. 